\title{
What is the spatial distribution of magnetic helicity injected in a solar active region? ${ }^{\star}$
}

\author{
E. Pariat ${ }^{1,2}$, A. Nindos $^{3}$, P. Démoulin ${ }^{1}$, and M. A. Berger ${ }^{4}$ \\ 1 LESIA, Observatoire de Paris-Meudon, UMR 8109 (CNRS), 92195 Meudon Cedex, France \\ e-mail: [etienne.pariat; pascal.demoulin]@obspm.fr \\ 2 Université Paris 7, Denis Diderot, 75251 Paris Cedex 05, France \\ 3 Section of Astrogeophysics, Department of Physics, University of Ioannina, 45110, Greece \\ e-mail: anindos@cc.uoi.gr \\ 4 Department of Mathematics, University College London, UK \\ e-mail: m.berger@ucl.ac.uk \\ Received 5 December 2005 / Accepted 26 February 2006
}

ABSTRACT

\begin{abstract}
Context. Magnetic helicity is suspected to play a key role in solar phenomena such as flares and coronal mass ejections. Several investigations have recently computed the photospheric flux of magnetic helicity in active regions. The derived spatial maps of the helicity flux density, called $G_{A}$, have an intrinsic mixed-sign patchy distribution.

Aims. Pariat et al. (2005) recently showed that $G_{A}$ is only a proxy of the helicity flux density, which tends to create spurious polarities. They proposed a better proxy, $G_{\theta}$. We investigate here the implications of this new approach on observed active regions.

Methods. The magnetic data are from MDI/SoHO instrument and the photospheric velocities are computed by local correlation tracking. Maps and temporal evolution of $G_{A}$ and $G_{\theta}$ are compared using the same data set for 5 active regions.

Results. Unlike the usual $G_{A}$ maps, most of our $G_{\theta}$ maps show almost unipolar spatial structures because the nondominant helicity flux densities are significantly suppressed. In a few cases, the $G_{\theta}$ maps still contain spurious bipolar signals. With further modelling we infer that the real helicity flux density is again unipolar. On time-scales larger than their transient temporal variations, the time evolution of the total helicity fluxes derived from $G_{A}$ and $G_{\theta}$ show small differences. However, unlike $G_{A}$, with $G_{\theta}$ the time evolution of the total flux is determined primarily by the predominant-signed flux while the nondominant-signed flux is roughly stable and probably mostly due to noise.

Conclusions. Our results strongly support the conclusion that the spatial distribution of helicity injected into active regions is much more coherent than previously thought: on the active region scale the sign of the injected helicity is predominantly uniform. These results have implications for the generation of the magnetic field (dynamo) and for the physics of both flares and coronal mass ejections.
\end{abstract}

Key words. Sun: magnetic fields - Sun: photosphere - Sun: Corona

\section{Introduction}

Magnetic helicity quantifies how the magnetic field is sheared and/or twisted compared to its lowest energy state, the potential field. Observations of the solar atmosphere show the existence of several sheared, even helical magnetic structures. Such structures are often associated with flares, eruptive filaments and coronal mass ejections (CMEs). Magnetic helicity thus appears as a key element in a large number of coronal phenomena and the computation of magnetic helicity is a very important task in solar physics. Therefore, it is not surprising that recently significant new developments have been made in the subject (see reviews in Brown et al. 1999; Berger 2003).

For a divergence-free field $\boldsymbol{B}$ within a bounded volume $\mathcal{V}$ of surface $\mathcal{S}$ where the normal component $B_{n}=\boldsymbol{B} \cdot \hat{\boldsymbol{n}}$ does not entirely vanish on $\mathcal{S}$ (i.e. like the situation in the solar corona), Berger \& Field (1984) have defined a relative magnetic helicity, $H . H$ is defined by subtracting the helicity of a reference field having the same normal component $B_{n}$ on $\mathcal{S}$ as $\boldsymbol{B}$. Using a

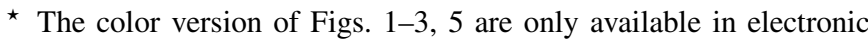
form at http: //www. edpsciences.org potential field, $\boldsymbol{B}_{p}$, is a convenient choice for a reference field. An expression for $H$, valid for any gauge has been given by Finn \& Antonsen (1985).

The direct computation of the helicity in the corona requires knowledge of the magnetic field in the entire volume, but measurements are mainly realized at the photospheric level; thus one of the best ways to estimate magnetic helicity is by integrating in time the helicity flux injected through the photosphere

\subsection{Helicity flux}

Using the gauge $\boldsymbol{\nabla} \cdot \boldsymbol{A}_{p}=0$, and selecting the boundary condition $\boldsymbol{A}_{p} \cdot \hat{\boldsymbol{n}}=0$ for the vector potential of the potential (reference) field, Berger \& Field (1984), derived the flux of magnetic helicity through the surface, in particular the solar photosphere:

$\frac{\mathrm{d} H}{\mathrm{~d} t}=2 \int_{\mathcal{S}}\left[\left(\boldsymbol{A}_{p} \cdot \boldsymbol{B}\right) v_{n}-\left(\boldsymbol{A}_{p} \cdot \boldsymbol{v}\right) B_{n}\right] \mathrm{d} \mathcal{S}$

where $v$ is the plasma velocity. The first term corresponds to the injection of magnetic helicity by advection (emergence) whereas the second term is the flux of helicity due to motions parallel to $\mathcal{S}$. 
In order to estimate $\mathrm{d} H / \mathrm{d} t$ from observations one should determine the velocity field $\boldsymbol{v}$ and the magnetic field $\boldsymbol{B}$ on the photosphere. Presently, the horizontal velocity field is derived by applying the local correlation tracking (LCT) method to follow explicitly the magnetic fluxes, using a time sequence of longitudinal magnetograms. Since only horizontal velocities are deduced from the temporal evolution of $B_{n}$, it has been believed that only the shear term could be derived (e.g. Chae et al. 2001; Nindos \& Zhang 2002; Moon et al. 2002b). However, when using the LCT method one estimates the flux tube velocity $\boldsymbol{u}$ parallel to $\mathcal{S}$, and not the plasma velocity, $\boldsymbol{v}$ : the velocity derived from the LCT method can be expressed as (Démoulin \& Berger 2003):

$\boldsymbol{u}=\boldsymbol{v}_{t}-\frac{v_{n}}{B_{n}} \boldsymbol{B}_{t}$,

and thus Eq. (1) becomes:

$\frac{\mathrm{d} H_{A}}{\mathrm{~d} t}=-2 \int_{\mathcal{S}}\left(\boldsymbol{A}_{p} \cdot \boldsymbol{u}\right) B_{n} \mathrm{~d} \mathcal{S}$,

where the subscript $A$ indicates that the flux is computed using the vector potential $\boldsymbol{A}_{p}$. As has been mentioned by Chae et al. (2004), we note that for a finite $v_{n} B_{t}$, Eq. (2) gives increasingly large velocity $u$ (like $1 / B_{n}$ ) as one gets closer to the inversion line. This large apparent velocity of the photospheric footpoints is present at the tip of an emerging flux tube, and it can largely be underestimated by the LCT algorithm. Thus a complementary method of measuring $v_{n}$ and $B_{t}$ in the vicinity of inversion lines is required. However, it is the finite product $\boldsymbol{u} B_{n}$ which enters into the helicity flux (Eq. (3)) so the singularities of $\boldsymbol{u}$ introduce a problem only in its measurement in the vicinity of the inversion line. In the ARs we have analyzed in this paper, there is no case where tendency of having large velocity $\boldsymbol{u}$ around $B_{n}=0$ is present.

\subsection{Helicity flux densities}

It appears natural to define a helicity flux density, $G_{A}$, as the integrand of Eq. (3):

$G_{A}(\boldsymbol{x})=-2\left(\boldsymbol{A}_{p} \cdot \boldsymbol{u}\right) B_{n}$.

$G_{A}$ has been used in several studies to determine the spatial injection patterns of magnetic helicity in active regions (e.g. Chae 2001; Chae et al. 2001, 2004; Kusano et al. 2002, 2004a,b; Maeshiro et al. 2005; Moon et al. 2002a,b, 2003a,b; Nindos $\&$ Zhang 2002; Nindos et al. 2003; Yamamoto et al. 2005; Yokoyama et al. 2003). In all these different works, $G_{A}$ maps always appear extremely complex both in space and time, with polarities of both signs present at any time.

However, in a recent work (Pariat et al. 2005) we showed that $G_{A}$ is not a real helicity flux density and that its properties introduce artificial polarities of both signs. If $G_{A}$ produces spurious signals, it is mostly due to the fact that helicity flux densities per unit surface are not physical quantities. Due to the properties of helicity, only helicity flux density per unit of elementary magnetic flux has a physical meaning (see the definition of $\mathrm{d} h_{\Phi} / \mathrm{d} t$ in Sect. 5). But to estimate such quantity using real observations, it is necessary to isolate flux tubes and determine their connectivity, which is actually not possible. Thus any definition of a helicity flux density will only be a proxy of the helicity flux density per unit magnetic flux - referred to as real helicity flux density in the following. But some definitions may have properties which do not permit us to estimate the injection patterns of magnetic helicity correctly.

In Pariat et al. (2005), we presented a new estimate of helicity flux density, $G_{\theta}$, that does not suffer from the same problems as $G_{A}$. The helicity flux can be understood as the quantity which measures how each magnetic polarity moves relatively to the others. It is possible to write the helicity flux as a double integral over the surface which involves the relative rotation rate, $\mathrm{d} \theta(\boldsymbol{r}) / \mathrm{d} t$, of pairs of photospheric positions defined by $\boldsymbol{x}$ and $\boldsymbol{x}^{\prime}$, with $\boldsymbol{r}=\boldsymbol{x}-\boldsymbol{x}^{\prime}$. For a planar surface one obtains:

$\frac{\mathrm{d} H_{\theta}}{\mathrm{d} t}=-\frac{1}{2 \pi} \int_{\mathcal{S}} \int_{\mathcal{S}^{\prime}} \frac{\mathrm{d} \theta(\boldsymbol{r})}{\mathrm{d} t} B_{n} B_{n}^{\prime} \mathrm{d} \mathcal{S} \mathrm{d} \mathcal{S}^{\prime}$,

where the subscript $\theta$ indicates that the flux is computed using the relative rotation rate. Then $G_{\theta}$ is simply defined by:

$G_{\theta}(\boldsymbol{x})=-\frac{B_{n}}{2 \pi} \int_{\mathcal{S}^{\prime}} \frac{\mathrm{d} \theta(\boldsymbol{r})}{\mathrm{d} t} B_{n}^{\prime} \mathrm{d} \mathcal{S}^{\prime}$

We compared the properties of $G_{A}$ and $G_{\theta}$, applying them to several theoretical models: translational motions of a single polarity, separation and rotation of two polarities, and emergence of a twisted flux tubes (Pariat et al. 2005). In all cases, we showed that $G_{A}$ almost always produces spurious signals that confuse the interpretation of the injection of helicity. $G_{\theta}$ produces also fake signals for some configurations. For example, let us consider an arcade formed of a single flux tube. Suppose that the footpoints rotate in opposite directions so that the tube rotates as a whole but does not increase its twist. Thus even if no helicity should be injected, $G_{\theta}$ polarities of opposite sign appear at each footpoint. The use of the helicity flux density per unit of elementary magnetic flux permits us to avoid these fake signals. However, generaly parasitic helicity flux density polarities are much fainter with $G_{\theta}$ than with $G_{A}$. In the case of the emergence of a twisted flux tube, we estimated that these spurious polarities mask the real injected helicity flux when the number of turns of the twisted flux tube is lower than a few tenths of a turn (the flux tube being represented by half a torus). With $G_{\theta}$ the threshold in the number of turns is ten times lower than with $G_{A}$.

\subsection{Aims of this paper}

From our previous theoretical analysis we concluded that $G_{\theta}$ is much better suited for determining the patterns of helicity injection. But is it true with real observations? In observations of $G_{A}$, are the patchy structures of positive and negative sign fake polarities induced by $G_{A}$ or are they real signals? Even if our theoretical analysis argues that $G_{\theta}$ is better, parameters such as the size of the magnetic polarities, the helicity they carry, the velocity of the photospheric motions involved in complex active regions, may be such that $G_{A}$ could account for the real injection of magnetic helicity. If not, one would observe different patterns between $G_{\theta}$ and $G_{A}$ maps. In such a case the obvious questions are: (1) can we understand the injection of helicity directly from $G_{\theta}$ maps? (2) does $G_{\theta}$ also produce fake polarities that mask the real injection pattern?

In order to address these questions, in this paper we compare $G_{A}$ and $G_{\theta}$ maps of the active regions (ARs) previously studied by Nindos et al. (2003). The data are described in Sect. 2. Section 3 presents the differences in the helicity density maps leading to a completely new view of the structure of the helicity injection in ARs. In Sect. 4 we compare the total helicity fluxes derived from the two different definitions of helicity flux density. Then we give examples of the limitations of $G_{\theta}$ (Sect. 5). Finally 
Table 1. Total helicity fluxes, $\mathrm{d} H_{A} / \mathrm{d} t$ (Eq. (3)) and $\mathrm{d} H_{\theta} / \mathrm{d} t$ (Eq. (5)) and minimum and maximum values of the helicity flux densities $G_{A}$ and $G_{\theta}$. Fluxes are in units of $10^{21} \mathrm{~Wb}^{2} \mathrm{~s}^{-1}$ and densities in units of $10^{6} \mathrm{~Wb}^{2} \mathrm{~m}^{-2} \mathrm{~s}^{-1}$.

\begin{tabular}{|c|c|c|c|c|c|c|c|}
\hline \multirow{2}{*}{$\begin{array}{c}\text { NOAA } \\
\text { AR }\end{array}$} & \multirow{2}{*}{$\begin{array}{c}\text { Date \& } \\
\text { Time (UT) }\end{array}$} & \multicolumn{2}{|c|}{ Total flux } & \multicolumn{2}{|c|}{$G_{A}$} & \multicolumn{2}{|c|}{$G_{\theta}$} \\
\hline & & $\mathrm{d} H_{A} / \mathrm{d} t$ & $\mathrm{~d} H_{\theta} / \mathrm{d} t$ & $\min$ & $\max$ & $\min$ & $\max$ \\
\hline \multirow[t]{2}{*}{8210} & $98 / 05 / 0209: 20$ & 15 & 14 & -69 & 74 & -11 & 42 \\
\hline & 98/05/03 21:55 & 8.9 & 13 & -134 & 125 & -16 & 49 \\
\hline \multirow[t]{2}{*}{8375} & 98/11/04 06:25 & 4.4 & 4.6 & -33 & 52 & -10 & 23 \\
\hline & 98/11/05 07:35 & 7.2 & 6.5 & -35 & 64 & -17 & 22 \\
\hline \multirow[t]{2}{*}{9114} & $00 / 08 / 08$ 03:35 & -2.9 & -2.4 & -26 & 27 & -7.1 & 5.6 \\
\hline & 00/08/09 14:20 & -4.2 & -4.3 & -27 & 18 & -16 & 5.5 \\
\hline \multirow[t]{2}{*}{9182} & $00 / 10 / 1021: 40$ & 3.8 & 4.8 & -21 & 38 & $\overline{-5.2}$ & 18 \\
\hline & 00/10/11 21:40 & 1.1 & 0.9 & -20 & 22 & -9.0 & 13 \\
\hline \multirow[t]{2}{*}{9201} & $00 / 10 / 22 \quad 12: 05$ & 0.15 & 0.12 & -8.1 & 9.1 & -3.1 & 4.2 \\
\hline & 00/10/25 09:30 & -0.63 & -0.68 & -8.8 & 3.5 & -1.8 & 0.8 \\
\hline
\end{tabular}

in Sect. 6 we present conclusions about the main implications of the re-interpretation of helicity injection pattern for the subphotospheric physics and coronal activity.

\section{Data and methods}

\subsection{Magnetic data}

We studied five active regions, namely AR 8210, AR 8375, AR 9114, AR 9182 and AR 9201. The details about these active regions, in particular the time evolution of their magnetic and velocity field as well as their eruptivity are presented in Nindos et al. (2003). In summary, all of them were associated with several major flares and CMEs. AR 8210, AR 8375 and AR 9114 were formed of one large concentrated magnetic polarity with the opposite polarity being much more dispersed. AR 9182 appeared as a bipolar active region and presented significant flux emergence near the leading spot. AR 9201 was a decaying active region, with both polarities decaying similarly. We used 1-min cadence and 96-min cadence MDI data. The 1-min cadence data were available at the time intervals indicated in Table 1 in Nindos et al. (2003). These data consist of both high-resolution images (pixel size of $0.6^{\prime \prime}$ ) and full-disk images (pixel size of $2^{\prime \prime}$ ). Since MDI magnetograms suffer from instrumental effects (Berger \& Lites 2003; Nindos \& Zhang 2002), we corrected all MDI fluxes as detailed in Nindos et al. (2003). Since we do not want to include solar rotation in the computations of helicity injection (which is in any case negligible, see Démoulin et al. 2002), we removed solar differential rotation, taking as reference time the time when the active region passes through the central meridian.

\subsection{Derivation of $G_{A}$}

In order to compute $G_{A}$ (Eq. (4)), three quantities must be derived from observations. First one needs to know $B_{n}$. The MDI data provide only the longitudinal (along the line of sight) component of the magnetic field and we assume that the photospheric magnetic field is vertical. The vertical field component is then directly equal to the longitudinal field divided by the cosine of the heliocentric angle of the active region. The estimation of the errors induced by this approximation is presented in Nindos et al. (2003).

Then the vector potential of the potential field, $\boldsymbol{A}_{p}$, needs to be computed. $\boldsymbol{A}_{p}$ is derived from $B_{n}$, following Chae (2001). In order to avoid boundary effects when using the fast Fourier transform, each original magnetogram has been placed at the center of a map which is $2-3$ times bigger, and $2048 \times 2048$ modes were used.
The last quantity which is required to compute $G_{A}$ is $\boldsymbol{u}$, the velocity of the footpoints of the flux tubes. $\boldsymbol{u}$ can be directly estimated by applying the LCT method to the longitudinal magnetograms. The errors and limitations induced by the LCT are discussed in Démoulin \& Berger (2003) and in Sect. 6 of Nindos et al. (2003). Here the LCT parameters used were $\omega=7.5^{\prime \prime}$ for the apodizing window function and $\Delta T=15-20 \mathrm{~min}$ for the time interval between a pair of images. Since LCT faces difficulties in regions such as sunspot umbrae, where the spatial variations of the field are small, we used white-light images and not the magnetograms to determine the velocity fields in the umbrae and penumbrae of sunspots.

\subsection{Derivation of $G_{\theta}$}

To compute $G_{\theta}$, we only need to know $B_{n}$ and $\boldsymbol{u}$. Since the vector potential is not required here, $G_{\theta}$ is not affected by the errors induced by the discrete fourier transform when computing $\boldsymbol{A}_{p}$. It is also not necessary to insert the data in a larger map. $G_{\theta}$ can be directly computed from the original data. Since Eq. (6) involves a double integral over the field of view, computing $G_{\theta}$ may be more CPU-time-consuming than computing $G_{A}$. This is why, when studying the evolution of the helicity flux in Sect. 4, we did not consider all magnetogram pixels to compute $\mathrm{d} H_{\theta} / \mathrm{d} t$. We only computed it at the pixels where the absolute value of the longitudinal field was higher than 20 Gauss. This allowed us to reduce significantly the computation time with only marginal influence on the computed values of $\mathrm{d} H_{\theta} / \mathrm{d} t$. Indeed for several cases, we checked that the differences between our results and the derived values when no threshold was used was less than a few percent.

At present no study has yet been done to estimate the influence of noise and systematic errors due to the LCT methods on $G_{\theta}$. The LCT method limitations adduced by Démoulin \& Berger (2003) should nevertheless influence any $G_{\theta}$ estimation. Even if this is an important issue and the subject of a future work, one should note that it is not important in our present study: our aim is here to compare $G_{\theta}$ and $G_{A}$ using the very same dataset.

\section{Helicity flux density maps}

Almost all $G_{A}$ maps presented in recent helicity studies show complex patterns with mixed polarities (Sect. 1.2). Kusano et al. (2002) concluded that in active regions positive and negative helicity is simultaneously injected, even in unipolar magnetic regions. However we claim here that this is mostly due to fake signals induced by $G_{A}$ which almost always mask the real injection of helicity. A more trustworthy helicity density, $G_{\theta}$, indeed 

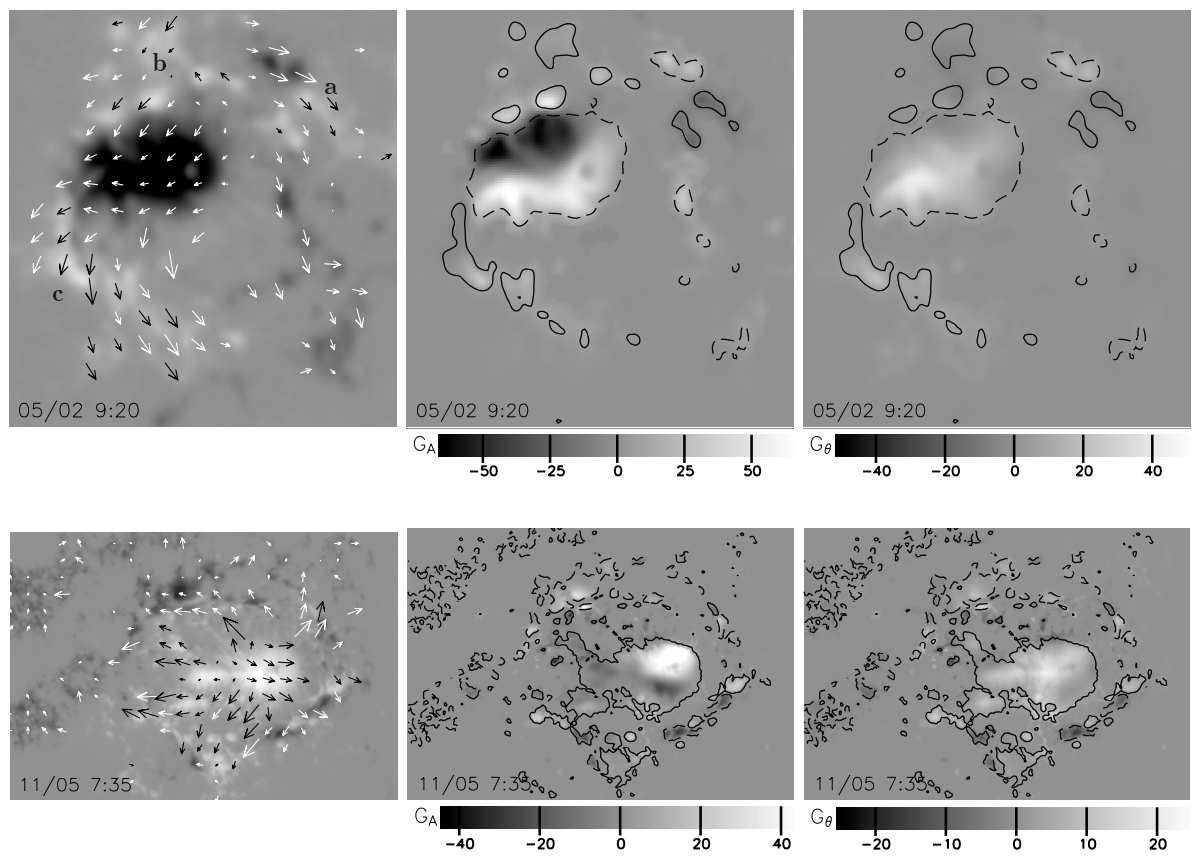

Fig. 1. AR 8210 at 09:20 UT on May 2, 1998. Left panel: $B_{n}$ magnetograms with velocity field (arrows). Center panel: $G_{A}$ maps. Right panel: $G_{\theta}$ maps. $G_{A}$ and $G_{\theta}$ maps are in units of $10^{6} \mathrm{~Wb}^{2} \mathrm{~m}^{-2} \mathrm{~s}^{-1}$ and have $\pm 300 \mathrm{G}$ isocontours of $B_{n}$. Note that the scale is not the same for the $G_{A}$ and the $G_{\theta}$ maps. (A color version with an additional example is available in the electronic version.)

Fig. 2. Same as Fig. 1 but for AR 8375 at 7:35 UT on November 5, 1998. (A color version with an additional example is available in the electronic version.) shows that the helicity injection patterns are rather uniform in sign. We will demonstrate that through several examples.

\subsection{Comparison for $A R 8210$}

AR 8210 has been the subject of several studies (see e.g. Welsch et al. 2004, and references therein) and produced several major flares and CMEs. It presents a $\delta$-configuration with a main negative-polarity spot (Fig. 1, right panel). This negative spot shows clockwise flow whereas anticlockwise motions dominate the positive polarities. Figure 1 presents $G_{\theta}$ and $G_{A}$ maps of AR 8210 on May 2, 1998, at 9:20 UT. The $G_{A}$ map is dominated by two polarities of opposite sign in the center of the negative magnetic spot. The surrounding positive magnetic polarities are associated with mostly positive injection of helicity. The maximum and minimum values reached by $G_{A}$ and $G_{\theta}$ are presented in Table 1 . The maximum absolute flux density is reached in the negative spot. Note that on May 3, the negative values of $G_{A}$ reach the strongest absolute values even if the total helicity flux is positive.

The $G_{\theta}$ map presents a completely different pattern than the $G_{A}$ map. The negative magnetic polarity here is almost entirely dominated by positive helicity flux density. There are no more strong negative helicity densities. The maximum value of the helicity flux density is thus much smaller in $G_{\theta}$ than in $G_{A}$. It is 1.7 times lower on May 2 and 2.6 times lower on May 3. The ratio $\min \left(G_{A}\right) / \min \left(G_{\theta}\right)$ is even more important. It is about 6.1 on May 2 and reaches 7.9 on May 3. Negative polarities become very faint in the $G_{\theta}$ map and thus the map is much more homogenous than the $G_{A}$ map.

The remnant negative helicity polarities could be real localized injection of negative helicity but could still be spurious signals, this time due to $G_{\theta}$. With our present data it is not possible to distinguish between these two possibilities; however with a closer analysis sometimes we may obtain some clues as we will show in Sect. 5. Whatever, these $G_{\theta}$ negative polarities have intensities lower than $1 / 5$ of those in $G_{A}$.

The surrounding magnetic polarities present fewer differences between $G_{A}$ and $G_{\theta}$ maps than the main polarity. Some regions have their helicity injection patterns unchanged, such as the bipole in the upper-right corner (noted $\mathbf{a}$ in Fig. 1). Other regions present opposite signs of helicity in $G_{A}$ and $G_{\theta}$ maps, for example the positive magnetic polarities which are north of the main magnetic polarity (noted $\mathbf{b}$ ). Some others regions, like the positive magnetic polarities below the main negative magnetic region (noted c), present the same characteristics as the main magnetic polarity: two opposite-sign polarity in $G_{A}$ and uniform positive injection in $G_{\theta}$. This complicates the interpretation: it is difficult to discriminate whether there is real injection or whether spurious signals are involved, both with $G_{A}$ and $G_{\theta}$. The intensities are nevertheless lower with $G_{\theta}$ than with $G_{A}$ in these areas, as in the main magnetic polarity.

\subsection{Other examples}

Similar patterns also appear in the other active regions. As an example in Fig. 2 we present for AR $8375 B_{n}$ magnetograms, $G_{A}$ and $G_{\theta}$ maps on November 5, 1998. Here the main positive magnetic polarity has a translational motion toward the solar west. The surrounding negative polarities show a divergent motion from the main spot. As in AR 8210, the main magnetic positive spot presents two polarities of $G_{A}$ with opposite signs whereas the $G_{\theta}$ maps show a more uniform pattern, primarily with positive helicity flux. Here also $G_{\theta}$ mainly reduces the nondominant flux densities (see minimum and maximum values of $G_{A}$ and $G_{\theta}$ in Table 1).

Figure 3 presents maps of the two helicity flux densities for AR 9114. Here again, $G_{A}$ maps present strong polarities of both signs while $G_{\theta}$ maps mostly present injection of negative helicity. For almost all the helicity flux density maps that we analysed - all are presented in Nindos et al. (2003) $-G_{\theta}$ maps appear much more uniform than $G_{A}$ maps. There are a few exceptions where strong polarities still remain in the $G_{\theta}$ map. But even for these few cases, the bipolar patterns are not located at the same place and have lower intensities than in $G_{A}$ maps. We will analyse in more detail such an example in Sect. 5. 

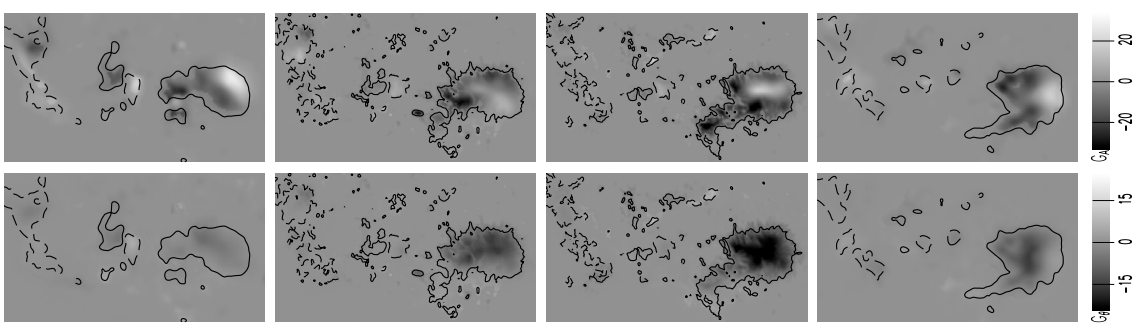

Fig. 3. $G_{A}$ (up) and $G_{\theta}$ (down) maps of AR 9114, as in Fig. 1. (A color version is available in the electronic version.)

\section{Comparison of $G_{A}$ and $G_{\theta}$ flux}

\subsection{Total fluxes}

Even if $G_{A}$ and $G_{\theta}$ do not have the same spatial properties, in theory the helicity flux integrated using $G_{A}, \mathrm{~d} H_{A} / \mathrm{d} t$ (Eq. (3)), and using $G_{\theta}, \mathrm{d} H_{\theta} / \mathrm{d} t$ (Eq. (5)) should be strictly equal, because both definitions are derived from Eq. (1).

However, when computing $\mathrm{d} H_{A} / \mathrm{d} t$ and $\mathrm{d} H_{\theta} / \mathrm{d} t$, some differences appear. For the $24 G_{A}$ maps of the 5 ARs that were presented by Nindos et al. (2003), we found a mean of 0.95 for the ratio between $\mathrm{d} H_{A} / \mathrm{d} t$ and $\mathrm{d} H_{\theta} / \mathrm{d} t$ derived using $G_{\theta}$. The mean absolute deviation of this ratio is equal to 0.25 . There is no systematic prevalence of one of these terms over the other and also no dependence on the sign of the helicity flux has been found. Concerning the data used in Fig. 4, we found that for the 1755 temporal values we considered, the mean ratio of $\mathrm{d} H_{A} / \mathrm{d} t$ to $\mathrm{d} H_{\theta} / \mathrm{d} t$ was around 0.94 with mean absolute deviation of 0.26 . If we consider the active regions separately we found that for AR 8210 the relative errors between $\mathrm{d} H_{A} / \mathrm{d} t$ and $\mathrm{d} H_{\theta} / \mathrm{d} t$ were of the order of a few per cent, while it was around $15 \%$ for AR 9114. Nevertheless we note that since there is no significant predominance of one of these two terms over the other, when we time-average the total flux evolution, the difference between $\mathrm{d} H_{A} / \mathrm{d} t$ and $\mathrm{d} H_{\theta} / \mathrm{d} t$ becomes very small. For example in AR 8210 the mean absolute deviation of the ratio $\mathrm{d} H_{A} / \mathrm{d} t$ over $\mathrm{d} H_{\theta} / \mathrm{d} t$ is 0.19 if one smooths the original data with 2 points and 0.10 if one uses 4 points.

A possible explanation for this discrepancy between these two active regions has to do with their mean helicity fluxes. AR 8210 has a mean absolute helicity flux several times larger than AR 9114 (see Fig. 4) although the magnetic flux of AR 8210 is lower than that of AR $9114\left(1.6 \times 10^{14}\right.$ and $3.1 \times$ $10^{14} \mathrm{~Wb}$ respectively). The differences between $\mathrm{d} H_{A} / \mathrm{d} t$ and $\mathrm{d} H_{\theta} / \mathrm{d} t$ tend to be smaller when the helicity flux involved is larger. This is probably the effect of noise in the data for the small flux values. Roughly, the differences tend to become important when the helicity flux is lower than $10^{21} \mathrm{~Wb}^{2} \mathrm{~s}^{-1}$. But these differences may also come from the larger errors in $\mathrm{d} H_{A} / \mathrm{d} t$ measurement due to the intense fake signals that $G_{A}$ produces.

\subsection{Signed fluxes}

Let us define the signed flux $\left(\mathrm{d} H_{A} / \mathrm{d} t\right)_{+}\left(\operatorname{resp} .\left(\mathrm{d} H_{A} / \mathrm{d} t\right)_{-}\right)$as the positive (resp. negative) flux of injected helicity, i.e. the sum of $G_{A}$ over the area where $G_{A}$ is $>0$ (resp. $<0$ ). Let us define $\left(\mathrm{d} H_{\theta} / \mathrm{d} t\right)_{+}$and $\left(\mathrm{d} H_{\theta} / \mathrm{d} t\right)_{-}$similarly but using $G_{\theta}$ instead of $G_{A}$ to integrate the flux.

The signed fluxes $\left|\left(\mathrm{d} H_{A} / \mathrm{d} t\right)_{ \pm}\right|$are always larger than $\left|\left(\mathrm{d} H_{\theta} / \mathrm{d} t\right)_{ \pm}\right|$, especially the flux whose sign is opposite to the total injected flux (see Fig. 4). For AR 8210 whose helicity flux is generally positive, $\left(\mathrm{d} H_{A} / \mathrm{d} t\right)_{+}$is in average 1.7 times larger than $\left(\mathrm{d} H_{\theta} / \mathrm{d} t\right)_{+}$and $\left|\left(\mathrm{d} H_{A} / \mathrm{d} t\right)_{-}\right|$is 3.0 times larger than $\left|\left(\mathrm{d} H_{\theta} / \mathrm{d} t\right)_{-}\right|$. The negative flux is strongly reduced when using $G_{\theta}$. In AR 9114
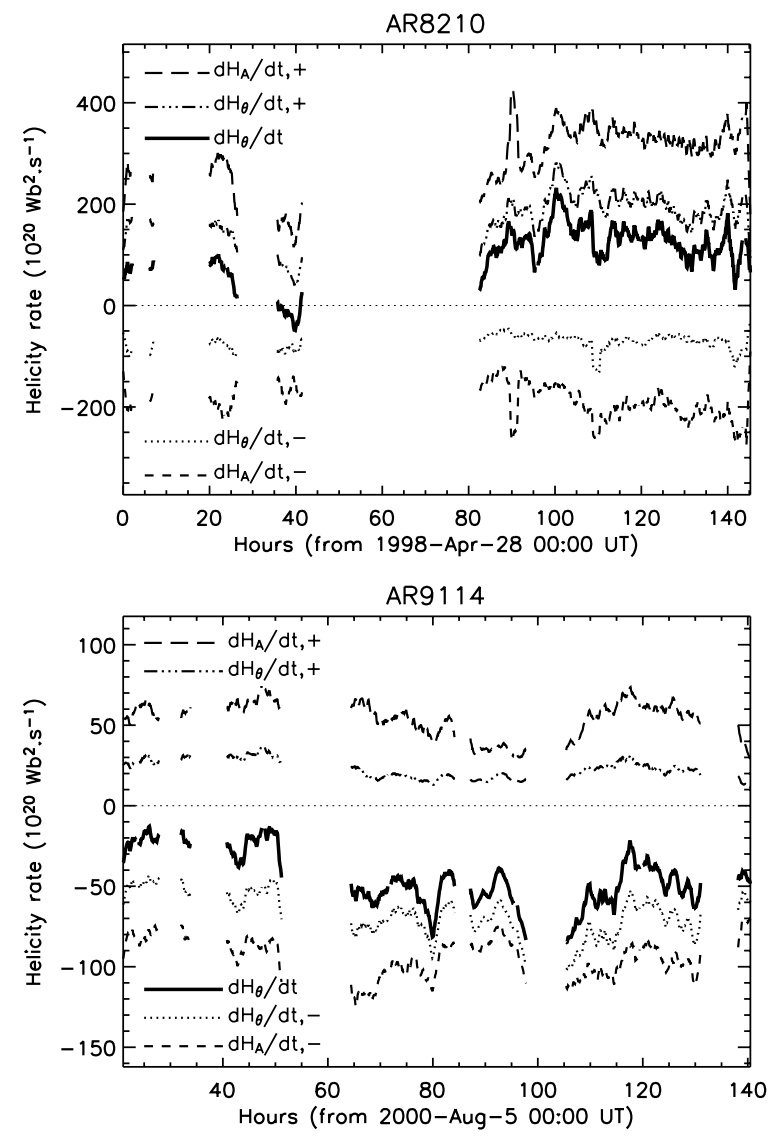

Fig. 4. Plots of $\left(\mathrm{d} H_{A} / \mathrm{d} t\right)_{ \pm},\left(\mathrm{d} H_{\theta} / \mathrm{d} t\right)_{ \pm}$and $\mathrm{d} H_{\theta} / \mathrm{d} t$ as a function of time for AR 8210 (up) and AR 9114 (down). The curves have been smoothed on a time interval of $100 \mathrm{~min}$. We do not present the $\mathrm{d} H_{A} / \mathrm{d} t$ curve because its differences with respect to the $\mathrm{d} H_{\theta} / \mathrm{d} t$ curve are too small to be clearly seen.

it is still the nondominant flux - the positive flux here - which becomes small when using $G_{\theta}$. Here $\left(\mathrm{d} H_{A} / \mathrm{d} t\right)_{+}$is on average 2.5 times larger than $\left(\mathrm{d} H_{\theta} / \mathrm{d} t\right)_{+}$and the mean ratio of $\left|\left(\mathrm{d} H_{A} / \mathrm{d} t\right)_{-}\right|$ over $\left|\left(\mathrm{d} H_{\theta} / \mathrm{d} t\right)_{-}\right|$is equal to 1.4 . In AR 9114 the ratios are smaller than in AR 8210 but this should be due to the fact that the total helicity flux is smaller in AR 9114 and so the noise in $G_{\theta}$ and the fake polarities induced by $G_{\theta}$ have a stronger influence.

With $G_{A}$, part of the strong fluxes of positive and negative helicity cancel out when summing over the whole surface, resulting in small total helicity fluxes. But it is not recommended to add quantities of opposite sign whose intensities are much larger than the intensity of the final result. The systematic errors tend to be added, resulting in a larger inaccuracy of the final result. Thus using $G_{\theta}$ should yield more accurate results when computing the total flux. 


\subsection{Time evolution of fluxes}

Another important feature with $G_{\theta}$ is that the study of the temporal evolution of helicity flux injection becomes much simpler. Figure 3 presents $G_{A}$ and $G_{\theta}$ maps at four different times. It is directly possible to follow qualitatively the evolution of the total helicity flux by visual inspection of the $G_{\theta}$ maps; for example in this case there is an increase of negative helicity and then a decrease. This evolution is not that apparent in the $G_{A}$ maps.

The fluctuations of the total and signed fluxes are also strongly reduced with $G_{\theta}$ compared to those with $G_{A}$. For example, in AR 9114, $\left(\mathrm{d} H_{A} / \mathrm{d} t\right)_{+},\left(\mathrm{d} H_{A} / \mathrm{d} t\right)_{-}$and $\mathrm{d} H_{A} / \mathrm{d} t$ present standard deviations of $3.5,7.3$, and $8.9 \times 10^{21} \mathrm{~Wb}^{2} \mathrm{~s}^{-1}$ respectively, while the standard deviations of $\left(\mathrm{d} H_{\theta} / \mathrm{d} t\right)_{+},\left(\mathrm{d} H_{\theta} / \mathrm{d} t\right)_{-}$, and $\mathrm{d} H_{\theta} / \mathrm{d} t$ are $0.8,1.6$, and $2.4 \times 10^{21} \mathrm{~Wb}^{2} \mathrm{~s}^{-1}$ respectively.

We also remark that with $G_{\theta}$, the nondominant flux is relatively constant (Fig. 4). For example in AR 8210 the mean and the standard deviation of $\left(\mathrm{d} H_{\theta} / \mathrm{d} t\right)_{-}$are respectively $-6.5 \times 10^{21} \mathrm{~Wb}^{2} \mathrm{~s}^{-1}$ and $2.0 \times 10^{21} \mathrm{~Wb}^{2} \mathrm{~s}^{-1}$ compared to $19.2 \times 10^{21} \mathrm{~Wb}^{2} \mathrm{~s}^{-1}$ and $6.5 \times 10^{21} \mathrm{~Wb}^{2} \mathrm{~s}^{-1}$ for $\left(\mathrm{d} H_{\theta} / \mathrm{d} t\right)_{+}$. This quasi-constant flux strongly questions the real origin of the nondominant signals. Possible reasons for the remnant nondominant helicity flux in $G_{\theta}$ maps could be the noise in the data and also the residual fake polarities that $G_{\theta}$ creates (see Sect. 5). Thus the intensity of these nondominant fluxes gives an idea of the accuracy on the total flux estimation: $\left\langle\left|\left(\mathrm{d} H_{\theta} / \mathrm{d} t\right)_{-}\right|\right\rangle=$ $6.5 \times 10^{21} \mathrm{~Wb}^{2} \mathrm{~s}^{-1}$ in $\mathrm{AR} 8210$ and $\left\langle\left(\mathrm{d} H_{\theta} / \mathrm{d} t\right)_{+}\right\rangle=2.1 \times$ $10^{21} \mathrm{~Wb}^{2} \mathrm{~s}^{-1}$ in AR 9114. These values are consistent with the fluctuations of the total helicity flux in non-flaring AR which was estimated to be about $3.2 \times 10^{21} \mathrm{~Wb}^{2} \mathrm{~s}^{-1}$ by Hartkorn \& Wang (2004) while the method is different.

Instead of the total net flux $\mathrm{d} H_{A} / \mathrm{d} t$, Maeshiro et al. (2005) have used the absolute flux (also called total unsigned flux), defined as the difference between the positive flux and the negative flux. They correlated the absolute helicity flux with X-ray activity. Nevertheless, most of the signals in $G_{A}$ are in fact spurious signals and are not linked to real helicity injection. Since $G_{A}$ produces stronger spurious signal when significant translatory motions are involved, the absolute helicity flux is a rather complex tracer of the photospheric field dynamics.

\section{Towards better helicity density maps}

\section{1. $G_{\theta}$ fake polarities}

In the previous examples, the $G_{\theta}$ fake polarities had small intensity compared to the main real helicity flux. However there are still some cases where the fake polarities of $G_{\theta}$ may remain dominant and corrupt the interpretation of the patterns of helicity flux. Figure 5 shows such an example.

Before October 9, 2000, AR 9182 consisted of a positive compact leading sunspot and a more extended trailing negative spot. From October 9, magnetic flux emergence occurs west of the active region, in the form of two separating oppositely-signed magnetic polarities (the evolution of this active region is presented in Fig. 7 of Nindos et al. 2003). The original leading sunspot is the positive magnetic polarity noted as $\mathrm{P}_{1}$ in Fig. 5 whereas the magnetic polarities of the emerging flux are indicated as $\mathrm{N}_{2}$ and $\mathrm{P}_{2}$. Since the AR was not close to disk center (N02 E46 on October 11), some longitudinal field reversal due to projection effects appear west of $\mathrm{P}_{2}$. We will not take that area into consideration.

The $G_{A}$ map (top left in Fig. 5) presents its usual complex patterns with several helicity flux polarities of both signs. In
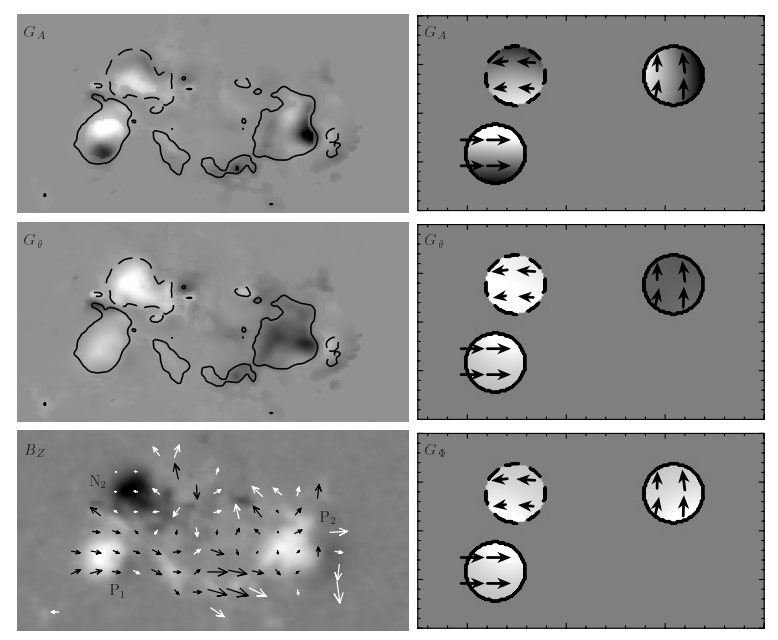

Fig. 5. Left row: AR 9182 on October 11, 2000 at 21:40 UT: $G_{A}$ map (top panel), $G_{\theta}$ map (middle panel) and $B_{n}$ magnetogram (bottom panel). The $B_{n}$ isocontours on the observations maps are for $B_{n}=$ \pm 300 G. Right row: model maps of helicity flux densities; $G_{A}$ (top panel), $G_{\theta}$ (middle panel) and $G_{\Phi}$ (bottom panel). (A color version is available in the electronic version.)

the $G_{\theta}$ map most of these patchy patterns have disappeared. However both positive and negative fluxes are very strong compared to the total flux and $G_{\theta}$ presents fluxes as strong as $G_{A}$, but their spatial distribution is different. Using the $G_{\theta}$ map, one would conclude that there is simultaneous injection of helicity of both signs in the emerging flux $\left(\mathrm{N}_{2} \mathrm{P}_{2}\right)$. But is this true?

\subsection{Defining a better flux density}

In Pariat et al. (2005), we found that only the helicity flux density per elementary flux tube (or per unit magnetic flux) could be defined. Let $\mathrm{d} h_{\Phi} /\left.\mathrm{d} t\right|_{e}$ denote the helicity injected in the elementary flux tube $e$ through its footpoints on the photosphere. Only this helicity flux per unit magnetic flux has a physical meaning.

Nevertheless, it is possible to represent this quantity as a helicity flux density per unit surface by distributing it between the footpoints of the elementary flux tube (whose positions are denoted as $\boldsymbol{x}_{e_{-}}$and $\boldsymbol{x}_{e_{+}}$). Each proxy for the helicity flux density is only a way of distributing $\mathrm{d} h_{\Phi} /\left.\mathrm{d} t\right|_{e}$, following some particular "rules". For example $\mathrm{d} h_{\Phi} /\left.\mathrm{d} t\right|_{e}$ can be related to $G_{A}$ with the relation:

$$
\begin{aligned}
\left.\frac{\mathrm{d} h_{\Phi}}{\mathrm{d} t}\right|_{e}= & \frac{1}{2}\left(\frac{G_{A}\left(\boldsymbol{x}_{e_{+}}\right)}{\left|B_{n}\left(\boldsymbol{x}_{e_{+}}\right)\right|}+\frac{G_{A}\left(\boldsymbol{x}_{e_{-}}\right)}{\mid B_{n}\left(\boldsymbol{x}_{e_{-}}\right)}\right) \\
& -\frac{1}{2 \pi} \int_{\mathcal{S}^{\prime}} \boldsymbol{u}^{\prime} \times\left(\frac{\boldsymbol{x}^{\prime}-\boldsymbol{x}_{e_{+}}}{\left(\boldsymbol{x}^{\prime}-\boldsymbol{x}_{e_{+}}\right)^{2}}-\frac{\boldsymbol{x}^{\prime}-\boldsymbol{x}_{e_{-}}}{\left(\boldsymbol{x}^{\prime}-\boldsymbol{x}_{e_{-}}\right)^{2}}\right) B_{n}^{\prime} \mathrm{d} \mathcal{S}^{\prime} .
\end{aligned}
$$

This equation is quite complex since $G_{A}$ fails to measure the net rotation of the dipole $\left(\boldsymbol{x}_{e_{+}} \boldsymbol{x}_{e_{-}}\right)$over the photospheric field. With $G_{\theta}$ the link is much more direct :

$\left.\frac{\mathrm{d} h_{\Phi}}{\mathrm{d} t}\right|_{e}=\frac{G_{\theta}\left(\boldsymbol{x}_{e_{+}}\right)}{\left|B_{n}\left(\boldsymbol{x}_{e_{+}}\right)\right|}+\frac{G_{\theta}\left(\boldsymbol{x}_{e_{-}}\right)}{\left|B_{n}\left(\boldsymbol{x}_{e_{-}}\right)\right|}$.

$\mathrm{d} h_{\Phi} /\left.\mathrm{d} t\right|_{e}$ is simply a field-weighted average of $G_{\theta}$ at both footpoints, and thus can be estimated using $G_{\theta}$ (provided that the field line connectivity is known).

If we suppose that the emerging polarity $\left(\mathrm{N}_{2} \mathrm{P}_{2}\right)$ in AR 9182 are still completely magnetically connected and that they form a single flux tube then the real helicity flux injected in this flux tube will be the sum of the helicity injected through $\mathrm{N}_{2}$ and $\mathrm{P}_{2}$. 
But since they have opposite values of $G_{\theta}$ with similar absolute intensities, the sign of $\mathrm{d} h_{\Phi} / \mathrm{d} t$ cannot be directly deduced. Even if intense signals of $G_{\theta}$ are present, the real injected helicity is much weaker. This is why here $G_{\theta}$ fails to give an accurate picture of the real patterns of injected helicity. For this particular case, the properties of $G_{\theta}$ distribute the helicity per unit magnetic flux over the footpoints in such a way that large fake polarities appear.

The best surface helicity flux density proxies of $\mathrm{d} h_{\Phi} / \mathrm{d} t$, can be obtained by sharing $\mathrm{d} h_{\Phi} / \mathrm{d} t$ equally between the two footpoints of each field line. One can use $G_{\Phi}$, defined as (derived from Eq. (29) of Pariat et al. 2005, with $f=1 / 2$ ):

$G_{\Phi}\left(x_{e_{ \pm}}\right)=\frac{1}{2}\left(G_{\theta}\left(\boldsymbol{x}_{e_{ \pm}}\right)+G_{\theta}\left(\boldsymbol{x}_{e_{\mp}}\right)\left|\frac{B_{n}\left(\boldsymbol{x}_{e_{ \pm}}\right)}{B_{n}\left(\boldsymbol{x}_{e_{\mp}}\right)}\right|\right)$.

\subsection{Helicity flux densities with a model}

In the ARs that we have analyzed it was not possible to determine the field line linkage. But in order to have an idea of what $G_{\Phi}$ would give for AR 9182, we can model this active region with two flux tubes having a torus shape with the same small radius and field strength. The distribution of the field perpendicular to the torus axis is assumed to be constant. The photospheric feet of the first flux tube corresponds to the original pre-existing polarity. The second half torus flux tube models the emerging magnetic flux $\left(\mathrm{N}_{2} \mathrm{P}_{2}\right)$. The model flux tubes are not twisted.

We implemented four kinds of motion for these polarities to describe the main velocity pattern observed in AR 9182. First, we considered a vertical emergence of the second flux tube. Second, we also imposed an eastward translational motion on that emerging flux tube, so that $\mathrm{P}_{2}$ does not present any eastwest motion. Third, we considered a solid rotation of this whole flux tube, relatively to $\mathrm{N}_{2}$. Finally, for the first flux tube, we only considered a translation toward the west. Observed and modelled $\boldsymbol{u}$ fields (Eq. (2)) are compared in Fig. 5. For simplicity, these torus-like flux tubes are represented when they are almost half-emerged, thus the sections of the tubes appear as circular regions, of opposite polarities.

We adjusted the relative intensities of the motions, in order to make a better correspondence between the observations and model, matching not only the velocity field but also the $G_{A}$ and $G_{\theta}$ patterns. With the above simple model we are able to derive both $G_{A}$ and $G_{\theta}$ having the same main features as the observed maps. In particular the main polarities of $G_{A}$ in AR 9182 are reproduced by the model. The model $G_{\theta}$ map, shows that negative density is located in $\mathrm{P}_{2}$ whereas positive helicity flux appears in $\mathrm{N}_{2}$ and $\mathrm{P}_{1} . \mathrm{N}_{2}$ presents the largest helicity flux, as in the observations.

The $G_{\Phi}$ map (bottom right in Fig. 5) gives a different result: the three magnetic polarities present positive helicity density. Among the evolution motions we considered, we found that the translational motion and the emergence are responsible for positive helicity injection while only the solid rotational motion of the emerging polarities is a source of negative helicity. With the considered relative intensities for each motion, the positive helicity dominates the helicity flux, the main source of helicity being the relative translational motions between $\left(\mathrm{N}_{1} \mathrm{P}_{1}\right)$ and $\left(\mathrm{N}_{2} \mathrm{P}_{2}\right)$.

Indeed mutual helicity is injected in both tubes due to the relative displacement of each tube with respect to the other. In particular the emergence of an untwisted flux tube nearby another one leads to injection of helicity in both tubes. This may appear paradoxical if one considers two untwisted closed rings of magnetic flux which are not linked. If there is no reconnection, no matter what kind of motions we apply to them their helicity should remain null. But when computing the relative helicity flux through a boundary which intersects these rings, one observes some helicity flux. This injection is mainly due to the apparent motion of the footpoints of the rising torus. Since the helicity flux changes sign when the torus is half emerged (the footpoint motion reverses), the total helicity injected will be null when the torus will be completely emerged, and so the paradox disappears.

If the emerging flux tube is twisted, the helicity flux due to the self-helicity may only appear if the twist is large enough to dominate this mutual helicity effect. In our model the mutual helicity injection will dominate if the number of turns for half the torus is lower than 0.002 , so only for very weakly twisted flux tubes. Since $G_{A}$ and $G_{\theta}$ patterns found for AR 9182 and for the model are qualitatively similar, this is an indication that the helicity flux density obtained for AR 9182 is dominated by the mutual helicity. Indeed, there is no evidence of twisting motions in the deduced LCT motions. We conclude that the LCT only detected the relative motions of the magnetic polarities (and may have missed some internal motions).

\section{Conclusion/discussion}

\subsection{Results}

In Pariat et al. (2005), we demonstrated with theoretical examples how the usual proxy of magnetic helicity flux density, $G_{A}$, can produce spurious signals, and we defined two new proxies of helicity flux density: $G_{\theta}$ and $G_{\Phi} . G_{\Phi}$ is the most accurate definition, but $G_{\theta}$ is practically more appropriate to work with observational data because $G_{\Phi}$ requires the knowledge of the field line connectivity. The present paper addresses the application of $G_{\theta}$ to real observations in comparison with $G_{A}$. We have applied $G_{\theta}$ to the same set of data that were studied by Nindos et al. (2003), in which $G_{A}$ was used.

The comparison of $G_{A}$ and $G_{\theta}$ maps shows that $G_{A}$ indeed creates strong fake polarities, due to the translational motions of magnetic polarities (Sect. 3). In general magnetic polarities of the same AR have different translational motions so it is not possible to remove a global motion to improve $G_{A}$ maps. With $G_{\theta}$ these spurious signal disappear: the nondominant polarities of the helicity flux are suppressed and the intensities of the predominant polarities are lowered. For all five active regions that we studied, the pattern of the helicity injection is much more homogeneous in $G_{\theta}$ maps than in $G_{A}$ maps. For most ARs, even if some nondominant polarities are still present in the $G_{\theta}$ maps, their intensities are much smaller than the intensities of the main helicity flux density polarities. Furthermore, it is not obvious whether these nondominant polarities are real signal, noise or spurious signal due to $G_{\theta}$.

The total and signed helicity flux computed using $G_{A}$ and $G_{\theta}$ were also compared. One expects that the total fluxes should be equal, but some differences do exist between $\mathrm{d} H_{A} / \mathrm{d} t$ and $\mathrm{d} H_{\theta} / \mathrm{d} t$ in our data (Sect. 4.1). Theses differences do not have a preferential sign and thus tend to become very small when the data are time-averaged. The relative differences are also somewhat stronger when the total fluxes are small in absolute value. Concerning the unsigned fluxes, the nondominant helicity flux with $G_{\theta}$ is strongly reduced compare with $G_{A}$ (Sect. 4.2). In the two active regions for which we computed the time evolution, the total flux is mainly due to the evolution of the dominant signed flux in $G_{\theta}$. The nondominant flux appears to be roughly 
constant. This may give an estimation of the intensities of the fake polarities that $G_{\theta}$ generates (Sect. 4.3) and an indication about the noise level in the computation of the total helicity flux.

Indeed, although $G_{\theta}$ reduces efficiently spurious signals induced by $G_{A}, G_{\theta}$ can also present fake polarities. AR 9182 is an example where $G_{\theta}$ creates intense unreal polarities (Sect. 5.1). In fact only the helicity flux density per elementary flux tube, $\mathrm{d} h_{\Phi} / \mathrm{d} t$, is physically meaningful and $G_{\Phi}$ is the best proxy for it (Sect. 5.2). It is nevertheless difficult to use such quantity since it is necessary to determine the coronal linkage to compute $G_{\Phi}$, which is presently not possible. In practice, $G_{\theta}$ is the best and simplest solution for mapping the injection of helicity. Even if it may induce spurious signals in some cases it is possible to infer the real patterns. For example, when two oppositely signed magnetic polarities which are believed to be linked present opposite $G_{\theta}$ signs, a better estimate of the real helicity flux is an average of the helicity densities at these magnetic polarities as shown in Fig. 5.

Regarding the pattern of the magnetic helicity injection we found that the helicity flux density distribution is much more homogeneous than previously thought. From the $28 G_{\theta}$ maps that we studied only 3 presented opposite sign polarities with intensities of the same magnitude. However in these cases, one may speculate that these patterns are formed primarily by fake polarities due to $G_{\theta}$ as we have demonstrated for AR 9182. We conclude that the scale for the helicity flux density polarities is at least of the order of the magnetic polarities if not of the scale of the whole active region.

\subsection{Implications of the results}

Meaningful statistics cannot come out from this work but the result of the homogeneous injection of magnetic helicity has implications in three domains.

The photospheric injection is a consequence of the generation of magnetic helicity in the solar interior and of its transport in the convection zone. The cyclonic convection ( $\alpha$-effect), which is thought to be the source of magnetic helicity of active regions, produces simultaneously positive and negative magnetic helicity but with a spectral segregation (Ji 1999). Brandenburg \& Blackman (2002) argue that magnetic helicity at different scales, and thus of different sign, must have different behaviors. The observed unipolar injection of magnetic helicity would define constraints for such models. However it is worth to keep in mind that presently the LCT method is efficient to detect motions of magnetic polarities (and mostly translatory motions) only on scales larger than the apodizing window. But is magnetic helicity flux homogeneous and of same sign at all scales? Refined methods to determine the velocity field are needed to improve the observational constraints; some are under development (Schuck 2005).

The injection of helicity with uniform sign has also some implications on some models of solar flare and CME triggering. Kusano et al. (2004b) developed a mechanism based on the annihilation of opposite-sign magnetic helicity. This model was motivated by the observations of mixed sign helicity injection. Even if one does not question the validity of their model, at least the scales and the frequencies for its application have to be studied. In order to test this model with observations it is important to remove as much as possible the fake polarities. Statistical studies of the helicity injection pattern - using $G_{\theta}$ and possibly $G_{\Phi}-$ linked to eruptive events must be performed.

Finally the injection of magnetic helicity with uniform sign supports the idea that CMEs are the way for the solar atmosphere to eject helicity (Rust 1994; Low 1996), and further that CMEs appear after sufficient amount of magnetic helicity has been stored (Nindos \& Andrews 2004). Regular, uniform, unipolar helicity flux through the photosphere slowly increases the absolute total coronal magnetic helicity of an active region, which will then have to eject it in CMEs.

Acknowledgements. A.N. acknowledges financial support from the Greek Ministry of Education's "Pythagoras II" grant.

\section{References}

Berger, M. A. 2003, Topological quantities in magnetohydrodynamics (Advances in Nonlinear Dynamics), 345

Berger, M. A., \& Field, G. B. 1984, J. Fluid Mech., 147, 133

Berger, T. E., \& Lites, B. W. 2003, Sol. Phys., 213, 213

Brandenburg, A., \& Blackman, E. G. 2002, in Solar Variability: From Core to Outer Frontiers, ESA SP-506, 805

Brown, M., Canfield, R., \& Pevtsov, A. 1999, Magnetic Helicity in Space and Laboratory Plasmas (Geophy. Mon. Ser. 111, AGU)

Chae, J. 2001, ApJ, 560, L95

Chae, J., Wang, H., Qiu, J., et al. 2001, ApJ, 560, 476

Chae, J., Moon, Y., \& Park, Y. 2004, Sol. Phys., 223, 39

Démoulin, P., \& Berger, M. A. 2003, Sol. Phys., 215, 203

Démoulin, P., Mandrini, C. H., van Driel-Gesztelyi, L., et al. 2002, A\&A, 382, 650

Finn, J. H., \& Antonsen, T. M. 1985, Comments Plasma Phys. Contr. Fusion, 9, 111

Hartkorn, K., \& Wang, H. 2004, Sol. Phys., 225, 311

Ji, H. 1999, Phys. Rev. Lett., 83, 3198

Kusano, K., Maeshiro, T., Yokoyama, T., \& Sakurai, T. 2002, ApJ, 577, 501

Kusano, K., Maeshiro, T., Yokoyama, T., \& Sakurai, T. 2004a, in ASP Conf. Ser., 175

Kusano, K., Maeshiro, T., Yokoyama, T., \& Sakurai, T. 2004b, ApJ, 610, 537

Low, B. C. 1996, Sol. Phys., 167, 217

Maeshiro, T., Kusano, K., Yokoyama, T., \& Sakurai, T. 2005, ApJ, 620, 1069

Moon, Y.-J., Chae, J., Choe, G. S., et al. 2002a, ApJ, 574, 1066

Moon, Y.-J., Chae, J., Wang, H., Choe, G. S., \& Park, Y. D. 2002b, ApJ, 580, 528

Moon, Y.-J., Chae, J., \& Park, Y. D. 2003a, J. Korean Astron. Soc., 36, 37

Moon, Y.-J., Chae, J., Wang, H., \& Park, Y. D. 2003b, Adv. Space Res., 32, 1953

Nindos, A., \& Andrews, M. D. 2004, ApJ, 616, L175

Nindos, A., \& Zhang, H. 2002, ApJ, 573, L133

Nindos, A., Zhang, J., \& Zhang, H. 2003, ApJ, 594, 1033

Pariat, E., Démoulin, P., \& Berger, M. A. 2005, A\&A, 439, 1191

Rust, D. M. 1994, Geophys. Res. Lett., 21, 241

Schuck, P. W. 2005, ApJ, 632, L53

Welsch, B. T., Fisher, G. H., Abbett, W. P., \& Regnier, S. 2004, ApJ, 610, 1148

Yamamoto, T. T., Kusano, K., Maeshiro, T., Yokoyama, T., \& Sakurai, T. 2005, ApJ, 624, 1072

Yokoyama, T., Kusano, K., Maeshiro, T., \& Sakurai, T. 2003, Adv. Space Res., 32,1949 
E. Pariat et al.: Helicity injection in a solar active region, Online Material p 1

\section{Online Material}


E. Pariat et al.: Helicity injection in a solar active region, Online Material p 2
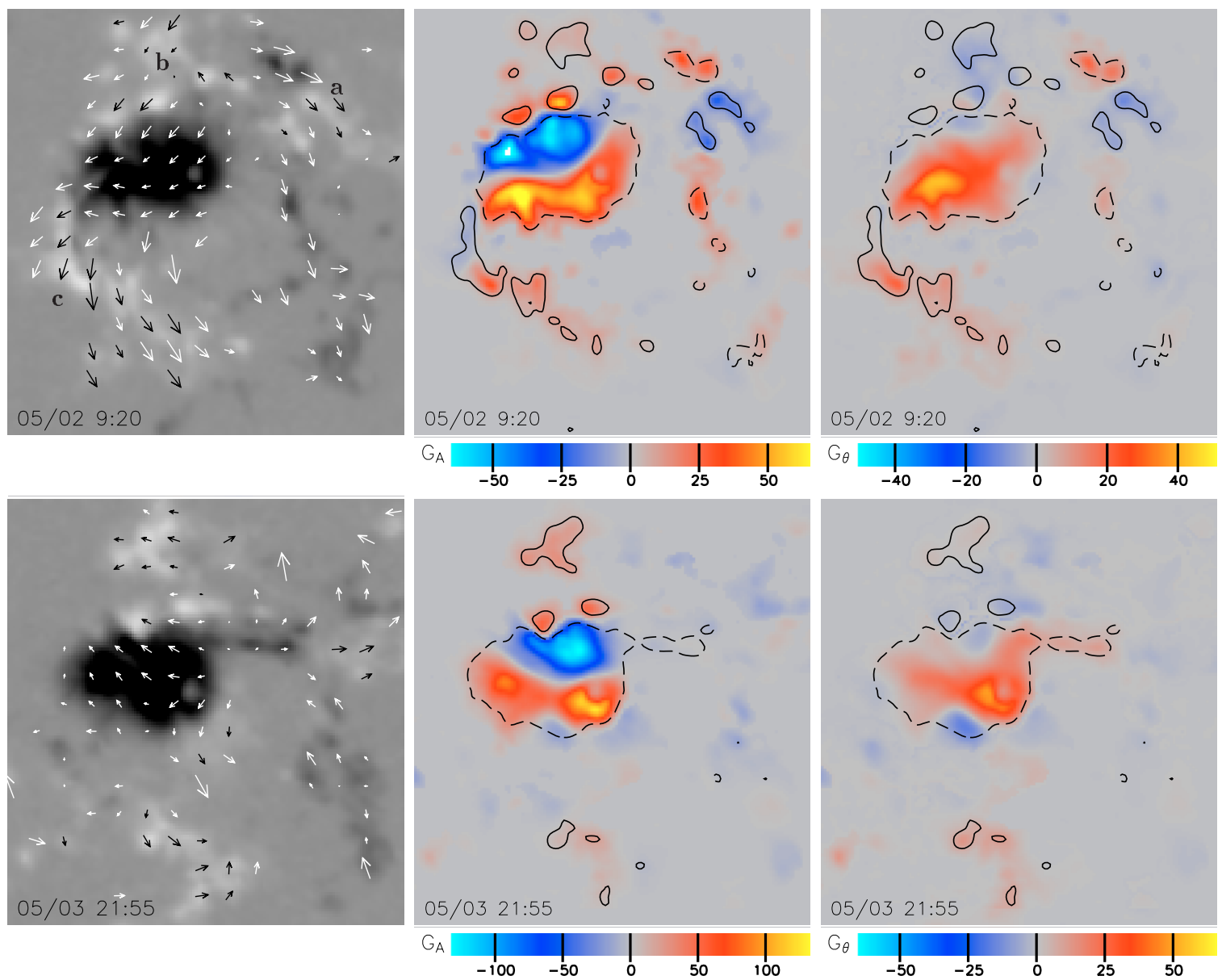

Fig. 1. AR 8210 at 09:20 UT on May 2, 1998 (top) and at 21:55 UT on May 3, 1998 (bottom). Left panels: $B_{n}$ magnetograms with velocity field (arrows). Center panels: $G_{A}$ maps. Right panels: $G_{\theta}$ maps. $G_{A}$ and $G_{\theta}$ maps are in units of $10^{6} \mathrm{~Wb}^{2} \mathrm{~m}^{-2} \mathrm{~s}^{-1}$ and have $\pm 300 \mathrm{G}$ isocontours of $B_{n}$. Note that the scale is not the same for the $G_{A}$ and the $G_{\theta}$ maps.
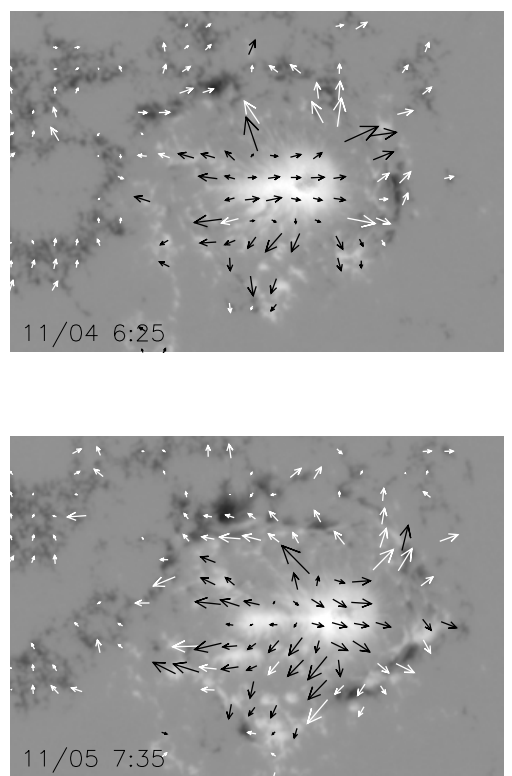
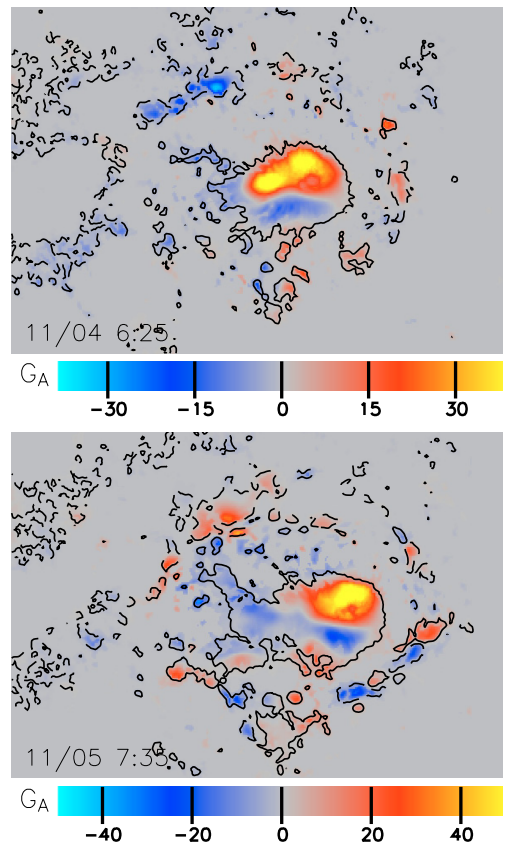
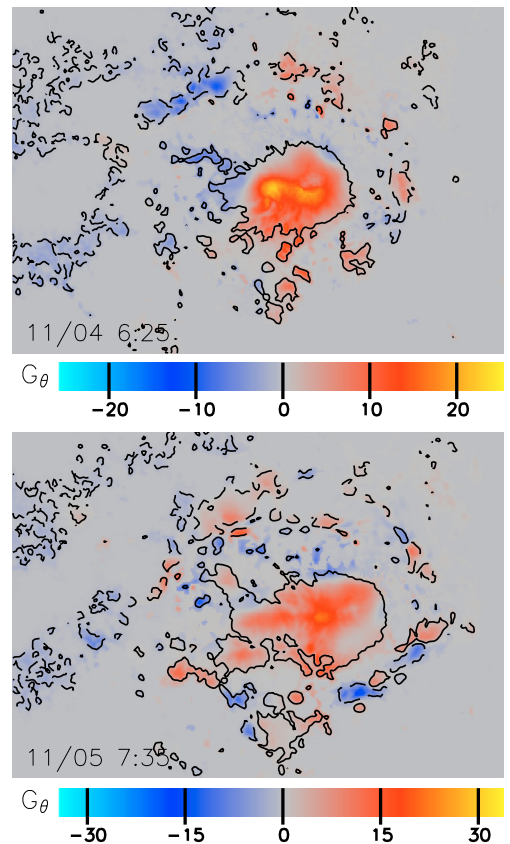

Fig. 2. Same as Fig. 1 but for AR 8375 at 06:25 UT on November 4, 1998 (top) and at 7:35 UT on November 5, 1998 (bottom). 
E. Pariat et al.: Helicity injection in a solar active region, Online Material $p 3$
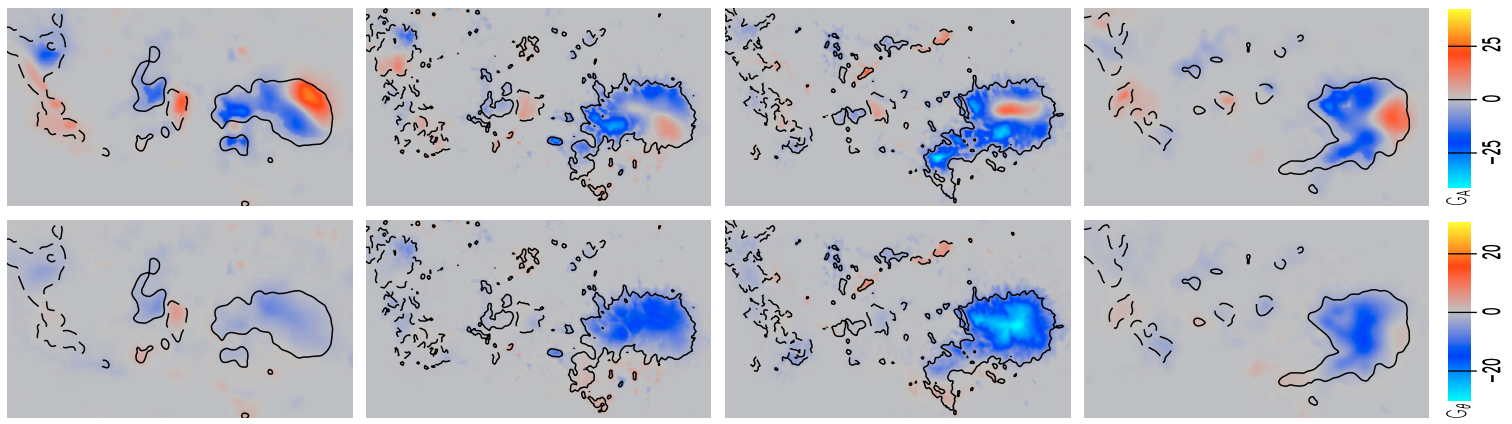

Fig. 3. Upper panel: plots of $\left(\mathrm{d} H_{A} / \mathrm{d} t\right)_{ \pm},\left(\mathrm{d} H_{\theta} / \mathrm{d} t\right)_{ \pm}$and $\mathrm{d} H_{\theta} / \mathrm{d} t$ as a function of time for AR 9114 . The curves have been smoothed on a time interval of $100 \mathrm{~min}$. We do not present the $\mathrm{d} H_{A} / \mathrm{d} t$ curve because its differences with respect to the $\mathrm{d} H_{\theta} / \mathrm{d} t$ curve are too small to be clearly seen. Middle panels and lower panels: $G_{A}$ and $G_{\theta}$ maps of AR 9114, as in Fig. 1.
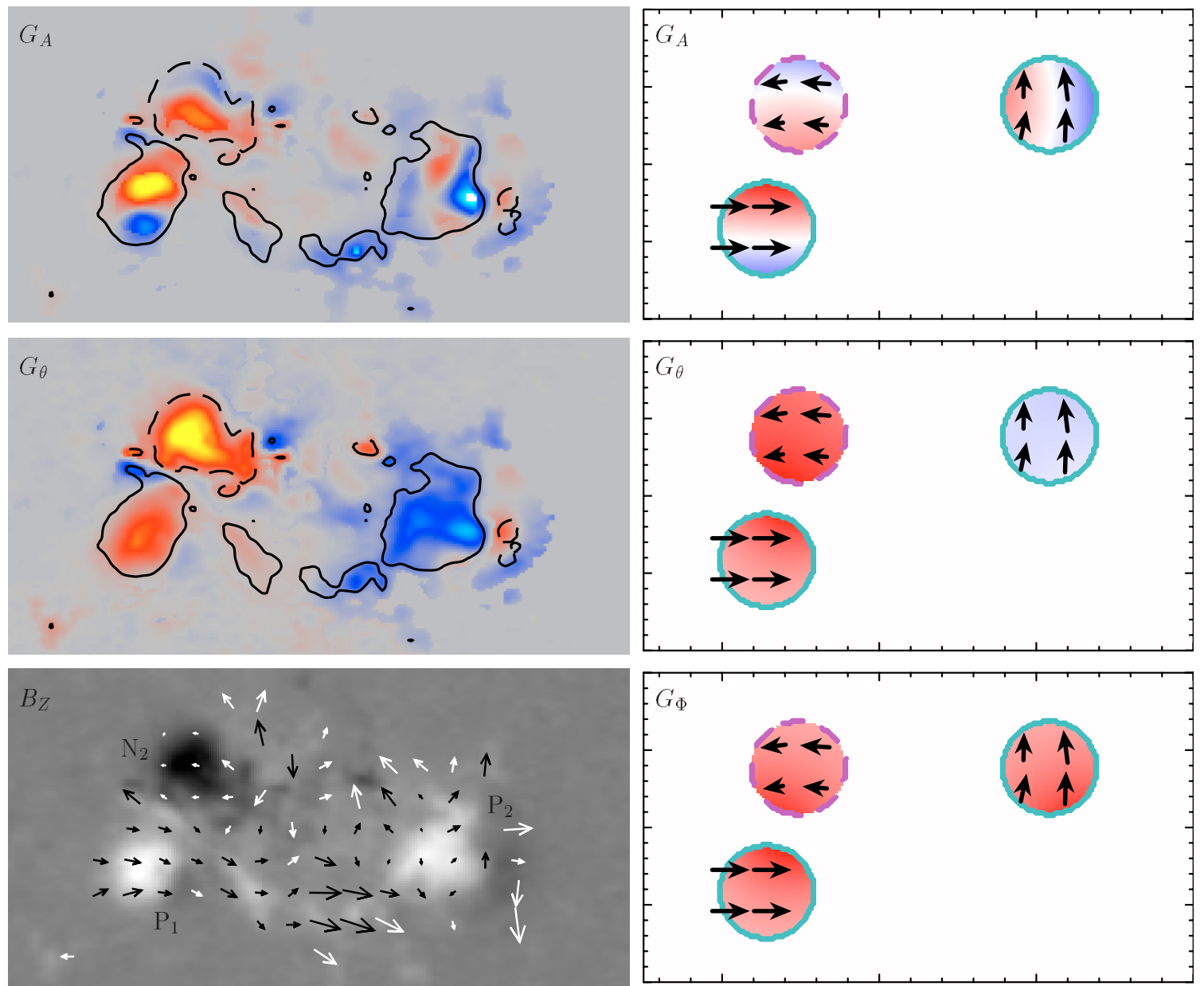

Fig. 5. Left row: AR 9182 on October 11, 2000 at 21:40 UT: $G_{A}$ map (top panel), $G_{\theta}$ map (middle panel) and $B_{n}$ magnetogram (bottom panel). The $B_{n}$ isocontours on the observations maps are for $B_{n}= \pm 300 \mathrm{G}$. Right row: model maps of helicity flux densities; $G_{A}$ (top panel), $G_{\theta}$ (middle panel) and $G_{\Phi}$ (bottom panel). 